Accepted for publication 12/10/98

\title{
ON THE PROFIT AND LOSS DISTRIBUTION OF DYNAMIC HEDGING STRATEGIES
}

\author{
SERGEI ESIPOV \\ Centre Solutions \\ a member of the Zurich Group \\ One Chase Manhattan Plaza \\ New York, NY 10005 \\ IGOR VAYSBURD \\ Martingale Technologies Inc \\ One Wall Street Court, Suite 300 \\ New York, NY 10005
}

\begin{abstract}
Hedging a derivative security with non-risk-neutral number of shares leads to portfolio profit or loss. Unlike in the Black-Scholes world, the net present value of all future cash flows till maturity is no longer deterministic, and basis risk may be present at any time. The key object of our analysis is probability distribution of future P\&L conditioned on the present value of the underlying. We consider time dynamics of this probability distribution for an arbitrary hedging strategy. We assume log-normal process for the value of the underlying asset and use convolution formula to relate conditional probability distribution of $\mathrm{P} \& \mathrm{~L}$ at any two successive time moments. It leads to a simple PDE on the probability measure parameterized by a hedging strategy. For risk-neutral replication the $\mathrm{P} \& \mathrm{~L}$ probability distribution collapses to a delta-function at the BlackScholes price of the contingent claim. Therefore, our approach is consistent with the Black-Scholes one and can be viewed as its generalization. We further analyze the PDE and derive formulae for hedging strategies targeting various objectives, such as minimizing variance or optimizing distribution quantiles. The developed method of computing the profit and loss distribution for a given hedging scheme is applied to the classical example of hedging a European call option using the "stop-loss" strategy. This strategy refers to holding 1 or 0 shares of the underlying security depending on the market value of such security. It is shown that the "stop-loss" strategy can lead to a loss even for an infinite frequency of re-balancing. The analytical method allows one to compute profit and loss distributions without relying on simulations. To demonstrate the strength of the method we reproduce the Monte Carlo results on "stop-loss" strategy given in Hull's book, and improve the precision beyond the limits of regular MonteCarlo simulations.
\end{abstract}




\section{Introduction}

Practitioners in financial markets rely on various extensions of the conventional BlackScholes (BS) analysis when basic BS assumptions do not hold. These assumptions require that the growth rate, risk-free rate of interest and volatility are constant, short selling of securities is permitted, transaction costs, taxes and dividends are absent, securities are perfectly divisible, trading is continuous, and there are no risk-less arbitrage opportunities ${ }^{[1]}$. Under these assumptions pricing reduces to replication, and there are no profits or losses (P\&L) associated with contracts. Attempts to overcome the restrictive assumptions inevitably involve some basis risk when the market participant is no longer certain regarding the present or future value of portfolios. Considerations of profit and loss can be sometimes suppressed by adjusting prices and implied volatilities. Pricing derivatives with stochastic rates and volatilities ${ }^{[2-4]}$, transaction costs ${ }^{[5,6]}$, hedging constraints ${ }^{[7-8]}$, and other realistic features lead to a designated price increase or decrease. It is supposed to compensate the financial institution for the residual basis risk.

As discussed below, it is practically impossible to eliminate the residual basis risk completely. Even if it were possible, investors might prefer innovative and risky strategies to risk-less replication of contingent claims. Such investors should be provided with a tool, which enables them to select appropriate hedging strategies and serve their personal risk profiles. Moreover, in incomplete markets risk can only be eliminated at a high cost ${ }^{[9-17]}$, and probabilistic approach seems to be the most natural one. With this in mind we suggest to consider this risk in detail, and focus on the probability distribution of profit and loss. Pricing in this respect becomes a separate issue, which should only be attempted after the P\&L distribution has been adequately described. In this paper the dynamics of the $\mathrm{P} \& \mathrm{~L}$ distribution is analyzed in conventional log-normal setting with the exception of Appendix A where general stochastic process is considered. Applications of this analysis to stop-loss strategy and other examples are discussed.

Pricing of basis risk is different in different markets. Insurance pricing corresponds to adding risk premium in proportion to cumulants or quantiles of the P\&L distribution. Credit derivatives are characterized by spread above risk-less rate. In this paper we only work with $\mathrm{P} \& \mathrm{~L}$ distributions, which precede pricing. After $\mathrm{P} \& \mathrm{~L}$ probability distribution is computed practitioners may refer to the guidelines of their financial institution to complete pricing.

Risk-neutral valuation is a general approach to studying derivatives ${ }^{[1]}$. It states that the price of a contract (European, for example), can be computed as an average of cash flow function at maturity over the equivalent risk-neutral martingale measure $M[S(t)]$ on a space of random walks of the underlying asset $[S(t)]$ together with an appropriate discounting

$$
F(S, t)=e^{r(t-T)} E_{\bar{M}}[F(\cdot, T)]
$$


This prescription follows from the no-arbitrage argument. The dot here denotes a realization of the random walk. The effective risk-neutral measure $\bar{M}$ comes as a result of gauging of an objective measure $M[S(t)]$ by the risk-less hedging strategy $\varphi_{0}(S, t)$

$$
\left(M, \varphi_{0}\right) \rightarrow \bar{M}
$$

Suppose a generic hedging strategy $\varphi \neq \varphi_{0}$ is chosen. This would lead to an effective measure $\bar{M}$ which is generally neither risk-neutral nor martingale. We shall solve the following problem. Given the objective measure $M$ (say, log-normal with drift $\mu$ and volatility $\sigma$ ) and a hedging function $\varphi$ derive an effective measure. As long as effective measure $\bar{M}$ is not risk-less, the value of future profit and loss is clearly uncertain. Therefore, we should describe $\bar{M}$ by a conditional probability distribution function (PDF) $P_{\varphi}(F \mid S, t)$ for a given underlying value, $S$, and time, $t$

$\int_{-\infty}^{\infty} d F P_{\varphi}(F \mid S, t)=1$

Then equation (1.1) takes more general form

$$
\left.P_{\varphi}(F \mid S, t)=e^{-r(t-T)} E_{\bar{M}_{\varphi}} \mid P\left(F e^{-r(t-T)} \mid \cdot, T\right)\right\rfloor .
$$

In the next Section we derive the partial differential equation for the time evolution of PDF and discuss it in detail beginning with a very basic case ${ }^{*}$. Section 3 is devoted to the equivalent martingale measure associated with PDE. Application of the developed methods to a simple example of the "stop-loss" strategy is given in Section 4. Optimal hedges are revisited in Section 5. Section 6 concludes the paper.

\section{Dynamics of Profit and Loss Distribution.}

\section{1 One Time Step}

To begin we consider a single-time (static) hedge position which starts at time zero and matures along with a European call option. Duration of the time step may be arbitrarily large. Let us again denote the P\&L value as $F$, underlying share value as $S$, number of shares of the underlying security used for hedging as $\varphi$, the portfolio value as $X$, time to maturity as $T$, option payoff at maturity as $F_{T}\left(S_{T}\right)$. The present best guess about the probability distribution of the share value at time $T$ is $p\left(S_{T}, T \mid S_{0}, 0\right)$, it is conditioned on today's price $S_{0}$. Let us consider a position consisting of one option and $\varphi$ shares. Such portfolio has a present value $X_{0}=F_{0}+\varphi S_{0}$. Making a one step in time to maturity one finds that the value of the portfolio, $X_{T}=F_{T}+\varphi S_{T}$, is uncertain because

\footnotetext{
${ }^{*}$ Part of this work has been reported previously ${ }^{[18,19]}$.
} 
of $S_{T}$ being uncertain. If the distribution $p\left(S_{T}, T \mid S_{0}, 0\right)$ is binary, with only two possible values, $S_{T}=S_{1}, S_{2}$, it is possible to suppress the variability of the portfolio $X_{T}$ by making use of the hedge ${ }^{[20]}$

$\varphi=-\frac{F_{1}-F_{2}}{S_{1}-S_{2}}$,

where $F_{1,2}=F_{T}\left(S_{1,2}\right)$. Given that $X_{T}=F_{T}+\varphi S_{T}=F_{1}+\varphi S_{1}=F_{2}+\varphi S_{2}$, the portfolio becomes deterministic. In view of the risk-free discounting for deterministic claims (perfectly replicated) one has $X_{0}=X_{T} e^{-r T}$, and this leads to a discrete Black-Scholes equation,

$F_{0}-F_{T} e^{-r T}+\varphi\left(S_{0}-S_{T} e^{-r T}\right)=0$.

Solution of (2.2) gives the option value ${ }^{[20]}$

$$
F_{0}=S_{0} \frac{F_{1}-F_{2}}{S_{1}-S_{2}}+e^{-r T} \frac{F_{2} S_{1}-F_{1} S_{2}}{S_{1}-S_{2}} .
$$

For an arbitrary distribution $p\left(S_{T}, T \mid S_{0}, 0\right)$ it is no longer possible to suppress the distribution of the portfolio. At the initial moment $t=0$ the PDF of the terminal portfolio value $X_{T}$ is simply expressed by the following convolution

$$
\tilde{P}\left(X_{T} \mid S, 0\right)=\int d S_{T} p\left(S_{T}, T \mid S_{0}, 0\right) \tilde{P}\left(X_{T}-\varphi S_{T} \mid S_{T}, T\right)
$$

Now suppose that a value of the portfolio at the moment $T$ is $X_{T}$ with some probability. Then clearly the $\mathrm{P} \& \mathrm{~L}$ value, as seen from the moment zero, is the discounted portfolio value minus initial investment, $F=X_{T} e^{-r T}-\varphi S$, with the same probability. This simple reasoning leads to the useful relation between the P\&L statistics at the moments 0 and $T$

$$
P\left(F \mid S_{0}, 0\right)=e^{r T} \int d S_{T} p\left(S_{T}, T \mid S_{0}, 0\right) P\left(\left(F+\varphi S_{0}\right) e^{r T}-\varphi S_{T} \mid S_{T}, T\right)
$$

An important lesson, which we learn from this exercise it that statistics of P\&L of cash flows is closely related to a hedging strategy. To find out how valuable a contract is one needs to examine it's statistical behavior under optimal hedging. We should return to this point below. 


\subsection{The Continuous Limit}

We now take the continuous limit assuming that the time step $t, t^{\prime}=t+d t$ is small and therefore, the log-normal kernel,

$$
p\left(S^{\prime}, t^{\prime} \mid S, t\right)=\frac{1}{S^{\prime} \sqrt{2 \pi \sigma^{2}\left(t^{\prime}-t\right)}} \exp \left\{-\frac{\left[\ln \left(S^{\prime} / S\right)-\left(\mu-\sigma^{2} / 2\right)\left(t^{\prime}-t\right)\right]^{2}}{2 \sigma^{2}\left(t^{\prime}-t\right)}\right\},
$$

is sharply peaked as compared to the typical support of $P(F \mid S, t)$. Then expanding the r.h.s. of (2.5) to the first order in $d t$ one finds ${ }^{*}[18,19]$

$$
\frac{\partial P}{\partial t}+r \frac{\partial}{\partial F}(F+\varphi S) P+\mu S\left(\frac{\partial P}{\partial S}-\varphi \frac{\partial P}{\partial F}\right)+\frac{1}{2} \sigma^{2} S^{2}\left(\varphi^{2} \frac{\partial^{2} P}{\partial F^{2}}-2 \varphi \frac{\partial^{2} P}{\partial F \partial S}+\frac{\partial^{2} P}{\partial S^{2}}\right)=0
$$

Second term on the 1.h.s. corresponds to continuous discounting of the portfolio, third term is responsible for the drift of the underlying and fourth one is a diffusion term a la Fokker-Plank. For the readers who prefer modern mathematical methods of stochastic calculus we present the alternative derivation in Appendix B. Eq(2.7) is supplied with a terminal condition. For a derivative security with payoff $F_{T}\left(S_{T}\right)$ the terminal condition is $P(F \mid S, T)=\delta\left[F-F_{T}(S)\right]$.

\subsection{Expected Values, Variances and Higher Cumulants}

The expected P\&L $\bar{F}(S, t)$ and its variance, $V(S, t)$

$$
\bar{F}(S, t)=\int d F F P(F \mid S, t), \quad V(S, t)=\int d F(F-\bar{F})^{2} P(F \mid S, t),
$$

obey partial differential equations, which can be easily derived from (2.7)

\footnotetext{
* This equation has been reported previously in Refs[18,19]. A mathematically similar equation arises in the study of passport options, Ref[23]. We are thankful to A. LiptonLifschitz for indicating this connection. The difference in the compounding rates in $\mathrm{Eq}(2.7)$ and $\mathrm{Eq}(13)$ of $\operatorname{Ref}[23]$ can be adjusted at the cost of dismissing risk-neutrality. The remaining difference is due to the fact that $\operatorname{Eq}(2.7)$ is written on the normalized probability density, while $\mathrm{Eq}(13)$ is written on the option value. Other forms of $\mathrm{Eq}(2.7)$ are given in Appendix B. One of these alternative forms (B.7) can be found in the presentation at the $8^{\text {th }}$ Annual Derivative Structures Conference (April 24-25, 1998, Boston University School of Management). This is the work by V. Putyatin and J. Dewynne "Mean-Variance Approach to Hedging European Option in the Presence of Transaction Costs". SE is thankful to S. Adamchuk for the reference.
} 


$$
\begin{aligned}
& \frac{\partial \bar{F}}{\partial t}+\mu S \frac{\partial \bar{F}}{\partial S}+\frac{1}{2} \sigma^{2} S^{2} \frac{\partial^{2} \bar{F}}{\partial S^{2}}-r \bar{F}=(r-\mu) \varphi S \\
& \frac{\partial V}{\partial t}+\mu S \frac{\partial V}{\partial S}+\frac{1}{2} \sigma^{2} S^{2} \frac{\partial^{2} V}{\partial S^{2}}-2 r V=\sigma^{2} S^{2}\left(\varphi+\frac{\partial \bar{F}}{\partial S}\right)^{2} .
\end{aligned}
$$

It is seen from $\mathrm{Eq}(2.9)$ that the variance of $\mathrm{P} \& \mathrm{~L}$ vanishes at $\varphi=-\partial \bar{F} / \partial S$. The $\mathrm{P} \& \mathrm{~L}$ becomes deterministic. It is nicely peaked at the BS value of the contingent claim since the substitution $\varphi=-\partial \bar{F} / \partial S$ in $\mathrm{Eq}(2.8)$ converts the latter into the BS equation,

$$
\frac{\partial \bar{F}}{\partial t}+r S \frac{\partial \bar{F}}{\partial S}+\frac{1}{2} \sigma^{2} S^{2} \frac{\partial^{2} \bar{F}}{\partial S^{2}}-r \bar{F}=0
$$

We briefly return to one time step considered in Subsection 2.1. From (2.5) one finds that the mean and variance of $F$ satisfy the following equations

$$
\begin{aligned}
& \bar{F}_{0}-e^{-r T} \bar{F}_{T}=\varphi S_{0}-e^{-r T} \varphi \bar{S}_{T} \\
& \operatorname{Var}\left(F_{0} \mid S_{0}\right)=e^{-2 r T}\left[\operatorname{Var}\left(F_{T}\right)-2 \varphi \operatorname{Cov}\left(F_{T}, S_{T}\right)+\varphi^{2} \operatorname{Var}\left(S_{T}\right)\right] .
\end{aligned}
$$

Eqs(2.11) and (2.12) require separate discussion for binary trees and other discrete settings. On binary trees, for every time step, one can define $\bar{F}_{1,2}$. At the nodes where the hedging strategy is $\varphi=-\left(\bar{F}_{1}-\bar{F}_{2}\right) /\left(S_{1}-S_{2}\right) \operatorname{Eq}(2.11)$ reduces to the $\mathrm{BS} \operatorname{Eq}(2.3)$ for the expected values of $\mathrm{P} \& \mathrm{~L}$, and the r.h.s. of (2.12) is zero in full agreement with the continuous case. For all other discrete settings the r.h.s. of (2.12) remains positive even for the variance-minimizing hedge

$\varphi=\operatorname{Cov}\left(F_{T}, S_{T}\right) / \operatorname{Var}\left(S_{T}\right)$,

and the equation for the expected value of $\mathrm{P} \& \mathrm{~L}$, which can be obtained by substituting (2.13) into (2.11), clearly deviates from the BS equation for the expected values of P\&L. Hedge (2.13) has been reported in ${ }^{[14]}$ along with the corresponding variance-optimal martingale measure. Formula (2.13) is well-known in finance as the optimal hedge ratio $\left(\mathrm{see}^{[1]} \mathrm{pp} 35-37\right)$.

We now return to the continuous case. The second-order operator in Eqs (2.7) is degenerate. This is a consequence of one Wiener process for two Eqs (2.1), (2.2). Introducing a function $\tilde{F}(S, t)$ defined as $\partial \tilde{F} / \partial S=-\varphi(S, t)$, and changing variable $F$ for $X=F-\tilde{F}(S, t)$, one finds from (2.7)

$$
\frac{\partial P}{\partial t}+r \frac{\partial(X P)}{\partial X}+\mu S \frac{\partial P}{\partial S}+\frac{1}{2} \sigma^{2} S^{2} \frac{\partial^{2} P}{\partial S^{2}}+\left(\frac{\partial \tilde{F}}{\partial t}+r S \frac{\partial \tilde{F}}{\partial S}+\frac{1}{2} \sigma^{2} S^{2} \frac{\partial^{2} \tilde{F}}{\partial S^{2}}-r \tilde{F}\right) \frac{\partial P}{\partial X}=0
$$


The reader can compare the term in parenthesis with $\mathrm{Eq}(2.8)$. Comparison shows that the basis risk is always present if the term in parenthesis is non-zero. This alternative form of the condition $\varphi=-\partial \bar{F} / \partial S$ (see above) is sufficient but it is not necessary if the final condition to $\mathrm{Eq}(2.14)$ already contains risk.

We conclude this Section with the equations for skewness, $S$, and kurtosis, $K$, defined for the P\&L distribution $P(F \mid S, t)$

$$
\mathrm{s}(S, t)=\int d F(F-\bar{F})^{3} P(F \mid S, t), \quad K(S, t)=\int d F(F-\bar{F})^{4} P(F \mid S, t)-3 V^{2}(S, t) .
$$

The equations read

$$
\begin{aligned}
& \frac{\partial s}{\partial t}+\mu S \frac{\partial s}{\partial S}+\frac{1}{2} \sigma^{2} S^{2} \frac{\partial^{2} s}{\partial S^{2}}-3 r s=3 \sigma^{2} S^{2} \frac{\partial V}{\partial S}\left(\varphi+\frac{\partial \bar{F}}{\partial S}\right) \\
& \frac{\partial K}{\partial t}+\mu S \frac{\partial K}{\partial S}+\frac{1}{2} \sigma^{2} S^{2} \frac{\partial^{2} K}{\partial S^{2}}-4 r K=\sigma^{2} S^{2}\left[-3\left(\frac{\partial V}{\partial S}\right)^{2}+4\left(\varphi-\frac{\partial \bar{F}}{\partial S}\right) \frac{\partial s}{\partial S}\right]
\end{aligned}
$$

Although the inhomogeneity quickly becomes cumbersome the structure of the differential operator on the 1.h.s. is very straightforward. We denote as $\hat{L}_{n}$ the Green function associated with this operator for a cumulant of order $n$

$$
\hat{L}_{n}\left(S^{\prime}, t^{\prime} \mid S, t\right)=\frac{e^{-n r\left(t^{\prime}-t\right)}}{S^{\prime} \sqrt{2 \pi \sigma^{2}\left(t^{\prime}-t\right)}} \exp \left\{-\frac{\left[\ln \left(S^{\prime} / S\right)-\left(\mu-\sigma^{2} / 2\right)\left(t^{\prime}-t\right)\right]^{2}}{2 \sigma^{2}\left(t^{\prime}-t\right)}\right\}
$$

It satisfies the backward equation

$$
\frac{\partial \hat{L}_{n}}{\partial t}+\mu S \frac{\partial \hat{L}_{n}}{\partial S}+\frac{1}{2} \sigma^{2} S^{2} \frac{\partial^{2} \hat{L}_{n}}{\partial S^{2}}-n r \hat{L}_{n}=\delta\left(S^{\prime}-S\right) \delta\left(t^{\prime}-t\right) .
$$

\section{The Equivalent Martingale Measure}

Presence of basis risk makes pricing by arbitrage uncertain. The risk-neutral equivalent martingale measure is no longer applicable. However, rewriting Eq(2.7) in the form

$$
\begin{aligned}
\frac{\partial P}{\partial t}+[r F-(r-\mu) \varphi S] & \frac{\partial P}{\partial F}+\mu S \frac{\partial P}{\partial S} \\
& +\frac{1}{2} \sigma^{2} S^{2}\left[\varphi^{2} \frac{\partial^{2} P}{\partial F^{2}}+2 \varphi \frac{\partial^{2} P}{\partial F \partial S}+\frac{\partial^{2} P}{\partial S^{2}}\right]=-r P,
\end{aligned}
$$


one finds that the left-hand-side of $\mathrm{Eq}(3.1)$ has the form of the backward Fokker-Planck equation, and corresponds to two coupled Itô stochastic differential equations

$$
\left\{\begin{array}{l}
d F_{\tau}=[r F+(\mu-r) \varphi S] d \tau+\sigma \varphi S d W, \\
d S_{\tau}=\mu S d \tau+\sigma S d W .
\end{array}\right.
$$

The joint distribution

$\mathrm{P}(F, S, T-t)=\left\langle\delta\left(F-F_{t}\right) \delta\left(S-S_{t}\right)\right\rangle$

generated by $\mathrm{Eq}(3.2)$ has to be discounted with the growth rate of underlying security to obtain the correct conditional probability density

$$
P(F \mid S, t)=e^{r(T-t)} \mathrm{P}\left(F e^{r(T-t)}, S, T-t\right) .
$$

Eqs (3.2), (3.4) define the equivalent martingale measure. We now proceed to computing the $\mathrm{P} \& \mathrm{~L}$ distributions for simple non-BS strategies.

\section{The "Stop-Loss" Strategy}

Believers of the "stop-loss" strategy assume that hedging the risk of derivatives is very straightforward ${ }^{[1,21]}$. For a long position in a European call option, which value at maturity is $\operatorname{Max}[S(T)-K, 0]=(S-K)^{+}$", the writer of the option is supposed to hold 1 share of the underlying asset when its market value is above $K$ and zero shares otherwise. Sometimes, a modified "stop-loss" is also used as a "put-replicating" investment strategy ${ }^{[22]}$.

The controversial aspect of the stop-loss strategy is the continuous limit ${ }^{[1,21]}$, where minimal time interval for re-balancing the portfolio, $\Delta t$, and the minimal step in quoted prices, $\Delta S$, are simultaneously taken to zero. The idea of "stop-loss" comes from the case when $\Delta S$ is taken to zero first. One can think that the re-balancing is done "immediately" at $S=K$.

For the "stop-loss" strategy one has $\varphi(S, t)=-\theta(S-K)$ where $\theta(x)$ is the Heaviside function. The variable $X$, see $\mathrm{Eq}(2.7)$ becomes $X=F+(S-K)^{+}$. This variable has the natural meaning of the total value, which consists of the value of the shares (if they are held) and the accumulated profit or loss, $F$.

The analytical solution of $\operatorname{Eq}(2.14)$ is given below for the simplest case $\mu=r=0$ and arithmetic Brownian motion (see also Appendix C). $\mathrm{Eq}(2.14)$ becomes

\footnotetext{
- $K$ is the strike. The option matures at $t=T$.
} 


$$
\frac{\partial P}{\partial t}+\frac{1}{2} \sigma^{2}\left(\delta(S) \frac{\partial P}{\partial X}+\frac{\partial^{2} P}{\partial S^{2}}\right)=0 ; \quad P(X \mid S, T)=\delta(X),
$$

where $\frac{1}{2} \sigma^{2}$ is now plays the role of diffusivity. One can see that the line $S=0$ is a very special place where, in the continuous limit, the portfolio loses money uniformly in time at a rate $\frac{1}{2} \sigma^{2}$. This is where the "stop-loss" strategy acquires quotation marks.

There exists an elegant method to solve $\mathrm{Eq}(4.1)$, which relies on mirror images. Here we give the most straightforward technique. An efficient solution by using integral transforms can be found in Appendix C. Using Green function of the diffusion equation the solution can be formally written as

$$
P(X \mid S, t)=\delta(X)+\frac{1}{2} \sigma^{2} \int_{t}^{T} d t^{\prime} \rho\left(S, t^{\prime}-t\right) \frac{\partial P\left(X \mid 0, t^{\prime}\right)}{\partial X}
$$

Denoting $f(X, t)=P(X \mid 0, t)$ we get a closed Volterra-type equation for $f(X, t)$

$$
f(X, t)=\delta(X)+\frac{\sigma}{\sqrt{8 \pi}} \int_{t}^{T} \frac{d t^{\prime}}{\sqrt{t^{\prime}-t}} \frac{\partial f\left(X, t^{\prime}\right)}{\partial X}
$$

The standard method of solving such equations is to consider (4.3) as an iterative recipe and find the resolvent. Denoting $f(X, t)=\delta(X) \sum_{n=0}^{\infty} C_{n}$ where

$$
C_{n+1}=1+\frac{\sigma}{\sqrt{8 \pi}} \int_{t}^{T} \frac{d t^{\prime}}{\sqrt{t-t^{\prime}}} \frac{\partial C_{n}}{\partial X},
$$

one can see that each integration adds a factor of $\frac{\sigma \sqrt{T-t} \Gamma\left(\frac{n}{2}+\frac{1}{2}\right)}{\sqrt{8} \Gamma\left(\frac{n}{2}+1\right)} \frac{d}{d X}$. As a result

$$
C_{n}=\left(\frac{\sigma \sqrt{T-t}}{\sqrt{8}} \frac{d}{d X}\right)^{n} \frac{1}{\Gamma\left(\frac{n}{2}+1\right)},
$$

and their sum is

$$
\sum_{n=0}^{\infty} C_{n}=\sum_{n=0}^{\infty}\left(\frac{\sigma \sqrt{T-t}}{\sqrt{8}} \frac{d}{d X}\right)^{n} \frac{1}{\Gamma(n / 2+1)}=\exp \left(\frac{\sigma^{2}(T-t)}{8} \frac{d^{2}}{d X^{2}}\right) \operatorname{erfc}\left(-\frac{\sigma \sqrt{T-t}}{\sqrt{8}} \frac{d}{d X}\right) .
$$


The function $f(X, t)$ is computed through its Fourier transform

$$
f=\int_{-\infty}^{\infty} \frac{d k}{2 \pi} \exp \left(-\frac{\sigma^{2} k^{2}(T-t)}{8}\right) \operatorname{erfc}\left(-\frac{i \sigma k \sqrt{T-t}}{\sqrt{8}}\right)=\frac{4 \theta(-X)}{\sqrt{2 \pi \sigma^{2}(T-t)}} \exp \left(-\frac{2 X^{2}}{\sigma^{2}(T-t)}\right) .
$$

It is interesting to note that this function can be expressed through the Green function of the diffusion equation, $f(X, t)=4 \theta(-X) \rho(2 X, t)$. One can see that at $S=0$, where function $f$ is defined, the probability evolves with a 4 times slower diffusivity then along $S$-axis. This is the slowest loss the "stop-loss" can achieve. Using (4.7) and returning to original variables one finds

$$
P(F \mid S, t)=\delta\left(F+S^{+}\right) \operatorname{erf}\left(\frac{|S|}{\sqrt{2 \sigma^{2}(T-t)}}\right)+\frac{4 \theta\left(-F-S^{+}\right)}{\sqrt{2 \pi \sigma^{2}(T-t)}} \exp \left[-\frac{(2 F+S)^{2}}{2 \sigma^{2}(T-t)}\right] .
$$

This answer consists of two parts. The first, with delta-function, refers to the scenarios where the underlying asset value never crosses the threshold $S=0$ (recall that here we measure $S$ from the strike price $K$ ). Other trajectories cross the threshold, and lead to losses. It is interesting to note that the answer given by $\mathrm{Eq}(4.8)$ is related to the local time distribution by virtue of the Tanaka formula (see ${ }^{[29]} \mathrm{p} .42$ ).

Another interesting case, which is reducible only to a convolution involving the Tanaka formula, arises when the stop-loss strategy is applied to a "zero payoff contract". In this case there is no payoff at maturity, and one is hedging zero. As before, 1 share is purchases every time the stock price is above the stop-loss benchmark. The relevant final condition is $P(F \mid S, T)=\delta(F)$ or $P(X \mid S, T)=\delta\left(X-S^{+}\right)$. We only give the final answer obtained by the method of mirror images. For $S \geq 0$

$$
P(F \mid S, t)=\theta(F+S)\left[\rho(F, T-t)-\frac{1}{3} \rho(F+2 S, T-t)\right]+\frac{8}{3} \theta(-F-S) \rho(2 F+S, T-t)
$$

and for $S<0$

$$
P(F \mid S, t)=\frac{2}{3} \theta(F) \rho(F-S, T-t)+\frac{8}{3} \theta(-F) \rho(2 F+S, T-t)+\delta(F) \operatorname{erf}\left(\frac{|S|}{\sqrt{2 \sigma^{2}(T-t)}}\right),
$$

where, as before, $\rho$ is the Green function of the diffusion equation.

We now return to the original log-normal case. A measure of the variability of the "stoploss" strategy, as suggested in ${ }^{[1]}$, is the ratio of the standard deviation defined by the probability distribution $P(F \mid S, t)$ to the Black-Scholes price of the perfectly hedged option. We gave a general expression for the variance of $P(F \mid S, t)$ in our previous 
work ${ }^{[19]}$, see Eqs $(10,11,21)$ in there. Since at the point $t=T$ the distribution is deterministic, the variance is given by the integral

$$
\begin{gathered}
V(S, t)=\sigma^{2} \int_{0}^{\infty} d S^{\prime} S^{\prime} \int_{t}^{T} \frac{d t^{\prime} e^{2 r\left(t-t^{\prime}\right)}}{\sqrt{2 \pi \sigma^{2}\left(t^{\prime}-t\right)}} \exp \left\{-\frac{\left[\ln \left(\frac{S^{\prime}}{S}\right)-\left(\mu-\frac{\sigma^{2}}{2}\right)\left(t^{\prime}-t\right)\right]^{2}}{2 \sigma^{2}\left(t^{\prime}-t\right)}\right\} \times \\
{\left[\theta\left(S^{\prime}-K\right)-\frac{\partial \bar{F}\left(S^{\prime}, t^{\prime}\right)}{\partial S^{\prime}}\right]^{2},}
\end{gathered}
$$

where the average profit or loss is

$$
\begin{aligned}
\bar{F}(S, t)=\int_{0}^{\infty} \frac{d S^{\prime}}{S^{\prime}} \int_{t} \frac{d t^{\prime} e^{r\left(t-t^{\prime}\right)}}{\sqrt{2 \pi \sigma^{2}\left(t^{\prime}-t\right)}} \exp \left\{-\frac{\left[\ln \left(\frac{S^{\prime}}{S}\right)-\left(\mu-\frac{\sigma^{2}}{2}\right)\left(t^{\prime}-t\right)\right]^{2}}{2 \sigma^{2}\left(t^{\prime}-t\right)}\right\} \times \\
{\left[S^{\prime} \theta\left(S^{\prime}-K\right)(r-\mu)+\delta\left(t^{\prime}-T\right)\left(S^{\prime}-K\right)^{+}\right] }
\end{aligned}
$$

Some of these integrals can be computed analytically, and the remaining work was done on Mathematica, using a set of parameters from the Hull's book ${ }^{[1]}$

$$
S=49, K=50, r=0.05, \sigma=0.20, T-t=0.3846, \mu=0.13 \text {. }
$$

The Black-Scholes price for the corresponding European call is $F=2.40047$. Our computation gave for the standard deviation of the profit or loss of the "stop-loss" strategy $V^{1 / 2}=1.8496 \pm 0.0002$. This fixes the ratio, $V^{1 / 2} / F=0.7705 \pm 0.0001$. In the Hull's book number 0.76 was obtained by a MC simulation with a finite re-balancing frequency. We challenge the readers with access to high-speed computers to reproduce our precision with Monte-Carlo simulations by performing of order $10^{8}$ iterations on very fine lattices.

\section{Realistic Optimal Hedges}

Hedging at discrete times, hedging with the subjective vlolatility and many other realistic strategies necessarily involve basis risk and can be optimized. If the strategy is fully specified, and it depends only on the value of the underlying asset and/or time, Eqs (2.4) - (2.9) can help to determine the residual basis risk and to control a chosen measure of risk. For example, discrete hedging at times $t=t_{m}, m=0,1,2, \ldots$ leads to the difference equations for the incremental contributions to cumulants [see $\operatorname{Eqs}(2.5),(2.6),(2.8),(2.9)]$ 


$$
\begin{aligned}
& \bar{F}_{m+1}-\bar{F}_{m}=(r-\mu) \varphi_{m} \hat{L}_{1}(S), \\
& V_{m+1}-V_{m}=\sigma^{2}\left[\varphi_{m}^{2} \hat{L}_{2}\left(S^{2}\right)-2 \varphi_{m} \hat{L}_{2}\left(S^{2} \partial_{S} \bar{F}\right)+\hat{L}_{2}\left(S^{2}\left(\partial_{S} \bar{F}\right)^{2}\right)\right], \\
& s_{m+1}-s_{m}=-3 \sigma^{2}\left\{\varphi_{m} \hat{L}_{3}\left(S^{2} \partial_{S} V\right)-\hat{L}_{3}\left[S^{2}\left(\partial_{S} \bar{F}\right)\left(\partial_{S} V\right)\right]\right\}, \\
& K_{m+1}-K_{m}=\sigma^{2}\left\{4 \varphi_{m} \hat{L}_{4}\left(S^{2} \partial_{S} s\right)-\hat{L}_{4}\left[3 S^{2}\left(\partial_{S} V\right)^{2}+4\left(\partial_{S} \bar{F}\right)\left(\partial_{S} s\right)\right]\right\} .
\end{aligned}
$$

where subscript $m$ refers to the moment $t_{m}$. Expressions under the Green function $\hat{L}_{n}$ are to be evaluated continuously by using Green functions of lower orders (see below). Increments of skewness and higher cumulants are linear functions of the number of shares of the underlying asset used for hedging, $\varphi_{m}$. Thus, as long as the range of acceptable hedge values is not artificially restricted, focusing on higher cumulants cannot be a part of the rational risk management scheme. Variance minimization, if attempted discretely requires, a hedge

$\varphi_{m}=\frac{\hat{L}_{2}\left(S^{2} \partial_{S} \bar{F}\right)}{\hat{L}_{2}\left(S^{2}\right)}$

This expression deviates from what can be found in literature ${ }^{[8]}$ although the vanishing leading order for small time steps is the same, and there is a clear resemblance to $\mathrm{Eq}(2.13) . \mathrm{Eq}(5.5)$ refers to a mixed discrete-continuous setting, where everything is continuous except for the process of rebalancing. In order to evaluate the right-hand-side of (5.5) and the resulting optimal variance, one has to solve (2.5) and (2.6) along with (5.5). The corresponding "continuous" hedge is simply $\varphi^{*}=\sum \varphi_{m} \theta\left(t-t_{m}\right) \theta\left(t_{m+1}-t\right)$. The solution for the expected profit and loss and corresponding variance is

$$
\begin{aligned}
\bar{F} & =(r-\mu) \hat{L}_{1}\left(S \varphi^{*}\right), \\
V & =\sigma^{2} \hat{L}_{2}\left[S^{2}\left(\varphi^{*}+\partial_{S} \bar{F}\right)^{2}\right] .
\end{aligned}
$$

Closed formulae can be obtained for the case of small time steps between the events of re-balancing, $\Delta t$. To the leading order in time step one finds from (5.6), (5.7)

$$
\begin{aligned}
& \sigma^{2} \hat{L}_{2}\left[S^{2}\left(\varphi^{*}+\partial_{S} \bar{F}\right)^{2}\right] \approx \sigma^{2} S^{2}\left(\frac{\partial^{2} \bar{F}}{\partial S^{2}}\right)^{2}\left\langle[S(t)-S(t+\Delta t)]^{2}\right\rangle \approx \frac{\Delta t}{2} \sigma^{4} S^{4}\left(\frac{\partial^{2} F}{\partial S^{2}}\right)^{2} \\
&(r-\mu) \hat{L}_{1}\left(S \varphi^{*}\right) \approx(\mu-r) S \frac{\partial \bar{F}}{\partial S}+(r-\mu) S \frac{\partial^{2} \bar{F}}{\partial S^{2}}\langle S(t)-S(t+\Delta t)\rangle \approx \\
& \approx(\mu-r) S \frac{\partial F}{\partial S}+\mu(\mu-r) S^{2} \frac{\partial^{2} F}{\partial S^{2}} \Delta t
\end{aligned}
$$


Here to the leading order one can replace $\bar{F}$ by $F$, the latter is the solution of the BS $\mathrm{Eq}(2.10)$, i.e. the option price for perfect replication. $\operatorname{Eqs}(2.8),(2.9)$ yield

$$
\begin{aligned}
& \frac{\partial G}{\partial t}+r S \frac{\partial G}{\partial S}+\frac{1}{2} \sigma^{2} S^{2} \frac{\partial^{2} G}{\partial S^{2}}-r G=\mu(\mu-r) S^{2} \frac{\partial^{2} F}{\partial S^{2}} \Delta t, \\
& \frac{\partial V}{\partial t}+\mu S \frac{\partial V}{\partial S}+\frac{1}{2} \sigma^{2} S^{2} \frac{\partial^{2} V}{\partial S^{2}}-2 r V=\frac{\Delta t}{2} \sigma^{4} S^{4}\left(\frac{\partial^{2} F}{\partial S^{2}}\right)^{2}
\end{aligned}
$$

where $G=\bar{F}-F$. Eqs(5.10), (5.11) are supplied with zero final conditions. $\operatorname{Eq}(5.11)$ has been reported ${ }^{[28]}$ (misprint $-\Delta t$ missing on the right-hand-side of $\mathrm{Eq}(5.3)$ of Ref[28]). Solutions of Eqs(5.10), (5.11) are easily expressed through the Green functions $\hat{L}_{1,2}$, respectively. In the case of a European call option with the strike $K$ the resulting integrals can be reduced to

$$
\begin{aligned}
& G=\Delta t \mu(\mu-r) K \frac{\sqrt{2(T-t)}}{\sigma \sqrt{\pi}} \int_{0}^{1} d \eta \exp \left[A_{1}-r \eta(T-t)\right] \\
& V=\Delta t \frac{(\sigma K)^{2}}{2 \pi} \int_{0}^{1} \frac{d \eta}{\sqrt{(1-\eta)(2-\eta)}} \exp \left[A_{2}-2 r \eta(T-t)\right]
\end{aligned}
$$

where

$$
\begin{aligned}
& A_{1}=\frac{1-\eta}{\eta} D(r)-\frac{1}{\eta} D\left(\frac{1}{2} \sigma^{2}\right)-C, \quad A_{2}=\frac{1-\eta}{\eta(2-\eta)} D\left(2 r-\frac{3}{2} \sigma^{2}\right)-\frac{1}{\eta} D\left(\frac{1}{2} \sigma^{2}\right)-2 C \\
& D(x)=\frac{[\ln (S / K)+(\mu-x)(T-t) \eta]^{2}}{2 \sigma^{2}(T-t)}, \quad C=\frac{1}{\sigma^{2}}\left(r+\frac{1}{2} \sigma^{2}\right)^{2}(T-t)(1-\eta) .
\end{aligned}
$$

Here it is assumed that $\Delta t$ is constant. (Variable $\Delta t(S, t)$ would remain under the integrals.) Recalling that by definition $G$ given by $\mathrm{Eq}(5.12)$ is the excess return "on top" of the BS price, and $V$ given by $\mathrm{Eq}(5.13)$ is the squared standard deviation of this excess return, we conclude that the excess return scales as the square of the standard deviation as the time step $\Delta t$ is taken to zero. This parabolic line in the plane excess return vs its standard deviation is clearly below any efficient frontier ${ }^{[30]}$ for small $\Delta t$ even if $G>0$. Thus, very frequent hedging or re-balancing is an inefficient investment of risk capital. The structure of the right-hand sides of Eqs(5.10), (5.11) implies that this statement holds for all options which can be perfectly replicated in theory. Formulae (5.12)-(5.14) can be used to get practical estimates of minimal reasonable time steps based on risk and return.

Different approach to risk management is to optimize the VaR or given quantile associated with the portfolio. Strictly speaking quantile hedging makes limited sense for a single contract, but may be of interest if the portfolio is large enough. One can show that holding 


$$
\varphi=\frac{(\mu-r) P+\sigma^{2} S \frac{\partial P}{\partial S}}{\sigma^{2} S \frac{\partial P}{\partial F}}
$$

securities represents the optimal hedge ${ }^{[19]}$. The expression (5.15) is evaluated at the quantile position which changes over time, its dynamics can be extracted from solving $\mathrm{Eq}(2.7)$ in parallel with (5.15).

\section{Conclusion}

We have presented a quantitative approach to the residual basis risk associated with dynamic hedging strategies. In the conventional log-normal setting evolution of the profit and loss distribution obeys a partial differential equation (2.7). By using this equation we have shown that the classical "stop-loss" strategy exhibits significant basis risk. The problem of discrete hedging in continuous time was visited briefly and the variance-minimizing solutions are given. Application of $\operatorname{Eq}(2.7)$ to contracts with discrete prices, and passport options ${ }^{[23]}$ is tempting, and this work is in progress. We remind that this study does not address pricing of residual risk ${ }^{[24]}$. Here we have only shown how to use the risk-minimizing replication to the best possible extent and access the remaining $\mathrm{P} \& \mathrm{~L}$ distribution, $P(F \mid S, t)$. When $P(F \mid S, t)$ is computed one may apply actuarial techniques (for recent interest in these techniques see ${ }^{[25]}$ ), methods used in credit markets, or VaR methods to handle the residual risk. Applying these methods from the very beginning, before the optimal replication is imposed, may lead to arbitrage.

\section{Acknowledgements}

The authors are grateful to John Hull and Peter Carr for interest and discussions, and to Marco Avellaneda for encouragement and suggestion to revisit the profit and loss of the "stop-loss" strategy using techniques presented here. Part of this work was written at Caffé Dante, and we thank their stuff for patience and understanding. SE is thankful to Dajiang Guo, Chunli Hou and Tim Newman for useful conversations. The authors acknowledge the interest by Iraj Kani and his invaluable help with the manuscript. Part of this work and its underlying philosophy was presented by one of us in the Fall of 1997 as a mini-course in the program entitled "Master of Arts in Mathematics" with specialization in the Mathematics of Finance at Columbia University (New York): http://www.math.columbia.edu/department/masters_finance.shtml. SE is grateful to Ioannis Karatzas and Jaksa Cvitani for their interest in insurance approach, to Jay Blumenstein for providing good communication with Columbia, discussions, and help while at Centre, and, certainly, to students of the program for their active participation and rewarding response. SE is indebted to the participants of the University of Chicago Financial Mathematics Seminar, where part of this work was presented, and to the Seminar organizer, Alexander Adamchuk, for warm hospitality.

All errors in this text are our responsibility. 


\section{References}

[1] J. Hull, Options, Futures and Other Derivatives, Prentice-Hall, Inc., $3^{\text {rd }}$ edition. (1997) 310312.

[2] J. C. Hull and A. White, The Pricing of Options on Assets with Stochastic Volatilities. Jouranl of Finance 42 (1987) 281-300

[3] S.I. Heston, A Closed Form Solution for Options with Stochastic Volatility with Applications to Bond and Currency Options, The Review of Financial Studies 6 (1993) 327-343

[4] D.S. Bates, Jumps and Stochastic Volatility: Exchange Rate Processes Implicit in Deutsche Mark Options, The Review of Financial Studies 9 (1996) 69-107.

[5] H.E. Leland, Option Pricing and Replication with Transaction Costs, Journal of Finance 40 n.5 (1985) 1283-1301

[6] K.B. Toft, Exact Formulas for Expected Hedging Error and Transaction Costs in Option Replication, UCB preprint (1993).

[7] J. Cvitani and I. Karatzas, Hedging Contingent Claims with Constrained Portfolios, Annals of Applied Probability 3 (1993) 652-681

[8] J. Cvitani, Optimal Trading under Contraints, in Financial Mathematics. W.J. Runggaldier (ed.), Lecture Notes in Mathematics \#1656, Springer, New York (1997)

[9] H. Föllmer and D. Sondermann, Hedging of Non-redundant Contingent Claims. In W. Hildenbrand and A. Mas-Colell (eds.) Contributions to Mathematical Economics (1986) 205223

[10] D. Duffie and H.R. Richardson, Mean-variance hedging in continuous time, Ann. Appl. Probab. 1 (1991) 1-15

[11] H. Föllmer and M. Schweizer, Hedging of Contingent Claims under Incomplete Information. In M.H.A. Davis and R.J. Elliott (eds.) Applied Stochastic Analysis, Stochastic Monographs, Gordon and Breach, London 5 (1991) 389-414

[12] M. Schweizer, Mean-Variance Hedging for General Claims, Annals of Applied Probability 2 (1992) $171-179$

[13] M. Schweizer, Approximating Random Variables by Stochastic Integrals, Annals of Probability 22 (1994) 1536-1575

[14] M. Schweizer, Variance-Optimal Hedging in Discrete Time, Mathematics of Operations Research 20 (1995) 1-32

[15] M. Schweizer, Approximation pricing and the Variance-Optimal Martingale Measure, Annals of Probability 24 (1996) 206-36

[16] J.P.Laurent and H.Pham, Dynamic Programming and Mean-Variance Hedging. (1997) preprint

[17] C.Hipp, Hedging and Insurance Risk. Preprint 1/96, University of Karlsruhe (1998)

[18] S. Esipov, unpublished (1996).

[19] S. Esipov and I. Vaysburd, Time Dynamics of Probability Measure and Hedging of Derivatives. Archive at Los Alamos (1998) http://xxx.lanl.gov/abs/math.PR/9805014

[20] J.C. Cox and M. Rubinstein, Options Markets, Prentice Hall, Englewood Hills, NJ, 1985.

[21] P. Carr, The Stop-Loss Start-Gain Strategy and Option Valuation, Review of Financial Studies, (1990)

[22] R.R. Trippi and R. B. Harriff, Dynamic Asset Allocation Rules: Survey and Synthesis, J. Portf. Mgmt, Summer (1991) 19

[23] T. Hyer, A. Lipton-Lifschitz, and D. Pugachevsky. Passport to Success, Risk, 10, No 9 (1997)

127-131. See also an extended version in the book Hedging with Trees, M. Broadie and P. Glasserman, eds., Risk Publications, London, Risk Books (1998) 49-56. 
[24] S.Esipov and D.Guo, Portfolio-Based Risk Pricing: Pricing Long-Term Put Options with GJR-GARCH(1,1)/Jump Diffusion Process, forthcoming paper in the Proceeding of the Seminar on Mathematical Finance at Courant Institute, World Scientific, Singapore (1998)

[25] The World According to Steve Ross, Derivatives Strategy, September (1998) 45-50

[26] C. W. Gardiner, Handbook of Stochastic Methods, $2^{\text {nd }}$ edition, Springer (1997)

[27] F. Russo and P.Vallois, Forward, backward and symmetric stochastic integration, Prob. Th. Rel. Fields 97 (1993) 403-421

[28] D. Bertsimas, L. Kogan, and A. Lo, When is Time Continuous?, preprint (1998)

[29] A. Borodin and P. Salminen, Handbook of Brownian Motion - Facts and Formulae, Birkhäuser Verlag, Basel, Boston, Berlin (1996).

[30]. H. M. Markowitz, Portfolio Selection, J. Finance, 7 (1952) 77

\section{Appendix A}

\section{General Stochastic Processes, Path Integral Solutions and The Effective Measure}

Deriving equation (2.7) we had in mind that the probability distribution is a smooth function of its arguments (otherwise partial derivatives would not be well defined or truncation of expansion at second order is problematic). Unfortunately, this is not always true. The PDE is not applicable if we have to deal with binary, delta-like or any other singular distribution at maturity. However, it is possible to derive closed form integral solution (evolution kernel) which would make sense for any reasonable final conditions $P(F \mid S, t)$.

Suppose that we are interested in PDF at the initial moment $t$. Let us divide the time interval $(t, T)$ in $N$ little segments $t_{k}, t_{k+1}$ such that $t_{0}=t$ and $t_{N}=T$. We should move backward in time - from maturity to the present moment - applying backward transfer matrix at each step. Introducing notations $S\left(t_{k}\right)=S_{k}$ and $\varphi\left(S, t_{k}\right)=\varphi_{k}$ one can rewrite $(2.5)$ as

$$
P\left(F \mid S_{k-1}, t_{k-1}\right)=\int d S_{k} T_{\varphi}\left(S_{k-1}, S_{k}\right) P\left(F \mid S_{k}, t_{k}\right)
$$

The $T$-operator acts on both arguments of $P$ - on $S$ as a trasfer-matrix and on $F$ as a shift. After $N$ successive backward steps we get

$$
P\left(F \mid S_{0}, t_{0}\right)=\int d S_{1} \ldots \int d S_{N} T_{\varphi}\left(S_{0}, S_{1}\right) \ldots T_{\varphi}\left(S_{N-1}, S_{N}\right) P\left(F \mid S_{N}, t_{N}\right)
$$

In the $N \rightarrow \infty$ limit the transfer-matrices accumulate to the measure

$$
\int D M_{\mid S(t)=S}=\int d S_{1} \ldots \int d S_{N} \rho\left(S_{0}, S_{1}\right) \ldots \rho\left(S_{N-1}, S_{N}\right)
$$

whereas stepwise shifts of $F$ produce an integral shift

$$
\Psi[S, \varphi]=\int_{t}^{T} e^{r\left(t-t^{\prime}\right)} \varphi(S, t)\left(d S_{t}-r S_{t} d t^{\prime}\right)
$$


c.f. (A.2) defined by the path $S_{t}$ and the hedging function $\varphi$. So, we get a compact answer for the PDF

$$
P(F \mid S, t)=e^{r(T-t)} \int D M[S] P\left(e^{r(T-t)}(F+\Psi[S, \varphi]) \mid \cdot T\right)
$$

Now it is appropriate to recall the formula (1.4). Effective measure $\bar{M}_{\varphi}$ on the space of random walks $S_{t}$ turns out to be a differential operator acting on probability

$$
\int D \bar{M}_{\varphi}[S]=\int D M[S] \exp \left(\Psi[S, \varphi] \partial_{F}\right)
$$

It should be stressed that formula (A.6) does not require any special properties of the probability measure like smoothness or continuity. It perfectly works for any distribution.

\section{Appendix B}

\section{Derivation of $\operatorname{Eq(2.7)~by~Stochastic~Calculus~}$}

Let it be again assumed (see (2.6)) that the underlying security $S(t)$ is described by a log-normal process in time $t, 0 \leq t \leq T$,

$d S_{t}=\mu S_{t} d t+\sigma S_{t} d W_{t}$

where the growth rate $\mu$ and volatility $\sigma$ are assumed to be constant, and the normalized Wiener process $W_{t}$ has zero mean and unit variance. If market participants follow a certain dynamic strategy $\varphi(S, t)$ for hedging, investment or portfolio insurance (which may differ from the perfect Black-Scholes hedge), the portfolio is risky and may generate profit and loss $X_{t}$ depending on realizations of the Wiener process in (B.1) and corresponding path $S(t)$ during the finite time interval $t \leq t^{\prime} \leq T$

$$
X_{[S], t, T}=\int_{t}^{T} e^{r\left(t-t^{\prime}\right)} \varphi(S, t)\left(d S_{t}-r S_{t} d t^{\prime}\right),
$$

where $r$ is the (constant) risk-free rate. Intuition behind $\mathrm{Eq}(\mathrm{B} .2)$ is based on a sequence of cash flows. During an infinitesimal time interval $d t^{\prime}$ the value of shares in the portfolio $\varphi S_{t}$ changed due to the change in asset value $\varphi S_{t} \rightarrow \varphi\left(S_{t}+d S_{t}\right)$ as seen "on top" of the risk-free appreciation $\varphi S_{t} \rightarrow \varphi\left(S_{t}-r S_{t} d t^{\prime}\right)$. The sum of these two independent changes discounted to present moment by the multiplier $e^{r\left(t-t^{\prime}\right)}$ is the "remaining" profit or loss of the portfolio. Differentiating (B.2) with respect to time one finds the stochastic process for the portfolio value 
$d X_{[S], t, T}=r X_{[S], t, T} d t-(\mu-r) \varphi\left(S_{t}, t\right) S_{t} d t+\sigma \varphi\left(S_{t}, t\right) S_{t} d W_{t}$,

In addition to future contribution to profit and loss (B.2) there exists P\&L accumulated from the past,

$$
X_{[S], 0, t}=\int_{0}^{t} e^{r\left(t-t^{\prime}\right)} \varphi(S, t)\left(d S_{t}-r S_{t} d t^{\prime}\right),
$$

which obeys Ito stochastic differential equation

$$
d X_{[S], 0, t}=r X_{[S], 0, t} d t+(\mu-r) \varphi\left(S_{t}, t\right) S_{t} d t+\sigma \varphi\left(S_{t}, t\right) S_{t} d W_{t} .
$$

It is noteworthy that $\mathrm{Eq}(\mathrm{B} .1)$ and, therefore, $\mathrm{Eq}(\mathrm{B} .5)$ are Ito stochastic differential equation (not Stratonovich). This property stems from the fact that the change of the asset value is assumed to be uncorrelated with the asset value and the number of shares $\varphi(S, t) . \mathrm{Eq}(\mathrm{B} .3)$ will be discussed below.

We first present forward and backward Fokker-Planck (FP) equations for (B.1), (B.5) ${ }^{[26]}$. The purely forward version reads

$$
\begin{aligned}
& \frac{\partial Q}{\partial T}=-\frac{\partial}{\partial X^{\prime}}\left[r X^{\prime}+(\mu-r) \varphi^{\prime} S^{\prime}\right] Q-\frac{\partial\left(\mu S^{\prime} Q\right)}{\partial S^{\prime}}+ \\
& \frac{1}{2} \sigma^{2}\left(\frac{\partial^{2}\left(\varphi^{\prime 2} S^{\prime 2} Q\right)}{\partial X^{\prime 2}}+2 \frac{\partial^{2}\left(\varphi^{\prime} S^{\prime 2} Q\right)}{\partial S^{\prime} \partial X^{\prime}}+\frac{\partial^{2}\left(S^{\prime 2} Q\right)}{\partial S^{\prime 2}}\right),
\end{aligned}
$$

while the purely backward version is

$$
\frac{\partial Q}{\partial t}+[r X+(\mu-r) \varphi S] \frac{\partial Q}{\partial X}+\mu S \frac{\partial Q}{\partial S}+\frac{1}{2} \sigma^{2} S^{2}\left(\varphi^{2} \frac{\partial^{2} Q}{\partial X^{2}}+2 \varphi \frac{\partial^{2} Q}{\partial S \partial X}+\frac{\partial^{2} Q}{\partial S^{2}}\right)=0 .
$$

Eqs (B.6) and (B.7) are equivalent, and describe the same joint probability density $Q\left(X, S, t \rightarrow X^{\prime}, S^{\prime}, T\right)$ to arrive at $S^{\prime}, X^{\prime}, T$ starting from $S, X, t$. The normalization condition reads

$\iint d S^{\prime} d X^{\prime} Q\left(X, S, t \rightarrow X^{\prime}, S^{\prime}, T\right)=1$.

(Obviously, the integral over $d S d X$ does not lead to any relevant constraint.)

In order to explain the difference between $\mathrm{Eq}(2.7)$ and regular FP equations (B.6), (B.7) we have to make a step back and address the issue of causality in detail. We shall show that the problem of contingent claims leads to an unusual stochastic calculus. 
The two classical conventions named after Ito and Stratonovich define the rules of stochastic integration for the so-called non-anticipating functions. From here on we follow very closely the textbook by Gardiner ${ }^{[26]}$ (see also ${ }^{[27]}$ ). Symbols $\partial / \partial x$ and $\partial_{x}$ are used interchangeably. Consider three stochastic integrals

$$
\begin{aligned}
& \mathrm{I} \int_{t_{0}}^{t} G\left[x\left(t^{\prime}\right), t^{\prime}\right] d W\left(t^{\prime}\right)=\underset{n \rightarrow \infty}{\operatorname{ms}-\lim } \sum_{i=1}^{n} G\left[x\left(t_{i-1}\right), t_{i-1}\right]\left[W\left(t_{i}\right)-W\left(t_{i-1}\right)\right], \\
& \mathrm{s} \int_{t_{0}}^{t} G\left[x\left(t^{\prime}\right), t^{\prime}\right] d W\left(t^{\prime}\right)=\underset{n \rightarrow \infty}{\operatorname{ms}-\lim } \sum_{i=1}^{n} G\left[\frac{1}{2} x\left(t_{i}\right)+\frac{1}{2} x\left(t_{i-1}\right), t_{i-1}\right]\left[W\left(t_{i}\right)-W\left(t_{i-1}\right)\right], \\
& \mathrm{z} \int_{t_{0}}^{t} G\left[x\left(t^{\prime}\right), t^{\prime}\right] d W\left(t^{\prime}\right)=\underset{n \rightarrow \infty}{\operatorname{ms}-\lim } \sum_{i=1}^{n} G\left[x\left(t_{i}\right), t_{i-1}\right]\left[W\left(t_{i}\right)-W\left(t_{i-1}\right)\right],
\end{aligned}
$$

where ms-lim stands for the so-called mean square limit in Hilbert space theory. Here $G(x, t)$ is an arbitrary function, and $W(t)$ is, again, a Wiener process. Convention (B.9) defines the Ito stochastic integral If , convention (B.10) defines the Stratonovich stochastic integral $s \int^{*}$, and (B.11) defines the integral $z \int$, which we analyze below.

It is possible to write a stochastic differential equation (SDE) using the latter integral

$$
x(t)=x\left(t_{0}\right)+\int_{t_{0}}^{t} d t^{\prime} A\left[x\left(t^{\prime}\right), t^{\prime}\right]+z \int d W\left(t^{\prime}\right) B\left[x\left(t^{\prime}\right), t^{\prime}\right],
$$

and find equivalent Ito SDE and Stratonovich SDE. Assuming that $x(t)$ is a solution of Ito SDE $d x=a d t+b d W$ one can deduce the corresponding $A$ and $B$. Using (B.11) and Ito's formula we write

$$
\begin{aligned}
& \mathrm{z} \int_{t_{0}}^{t} B\left[x\left(t^{\prime}\right), t^{\prime}\right] d W\left(t^{\prime}\right)=\underset{n \rightarrow \infty}{\operatorname{ms}-\lim } \sum_{i=1}^{n} B\left[x\left(t_{i-1}\right)+d x\left(t_{i-1}\right), t_{i-1}\right]\left[W\left(t_{i}\right)-W\left(t_{i-1}\right)\right]= \\
& \underset{n \rightarrow>\infty}{\operatorname{ms}-\lim } \sum_{i=1}^{n}\left\{B_{i-1}+\left[a_{i-1} \partial_{x} B_{i-1}+\frac{1}{2} b_{i-1}^{2} \partial_{x}^{2} B_{i-1}\right]\left(t_{i}-t_{i-1}\right)+\right. \\
& \left.b_{i-1} \partial_{x} B_{i-1}\left[W\left(t_{i}\right)-W\left(t_{i-1}\right)\right]\right\}\left[W\left(t_{i}\right)-W\left(t_{i-1}\right)\right]= \\
& \mathrm{I} \int_{t_{0}}^{t} B\left[x\left(t^{\prime}\right), t^{\prime}\right] d W\left(t^{\prime}\right)+\int_{t_{0}}^{t} d t^{\prime} b\left[x\left(t^{\prime}\right), t^{\prime}\right] \partial_{x} B\left[x\left(t^{\prime}\right), t^{\prime}\right]
\end{aligned}
$$

\footnotetext{
* The symbols $s \int$ appear together and should not be confused with the skewness $s$ defined in the main text.
} 
where, for brevity, we write $B_{i}$ etc, instead of $B\left[x\left(t_{i}\right), t_{i}\right]$. This formula gives a connection between our integral and the Ito integral. We observe that the Ito SDE

$d x=a d t+b d W$,

is the same as our $\mathrm{SDE}$

$d x=\left(a-b \partial_{x} b\right) d t+b d W$,

and (is known to be) the same as the Stratonovich SDE

$d x=\left(a-\frac{1}{2} b \partial_{x} b\right) d t+b d W$.

Conversely, our SDE

$d x=A d t+B d W$,

(B.17)

is the same as the Ito SDE

$d x=\left(A+B \partial_{x} B\right) d t+B d W$,

and the same as the Stratonovich SDE

$d x=\left(A+\frac{1}{2} B \partial_{x} B\right) d t+B d W$.

Our SDE defines a special calculus, which is different from ordinary calculus in the same manner as the Ito calculus. Namely, making a connection between (B.17) and (B.18), changing variables, using Ito's formula, and translating back with the help of (B.14) $\rightarrow$ (B.13), we find

$d f(x)=\left(\frac{\partial f}{\partial x} A-\frac{1}{2} \frac{\partial^{2} f}{\partial x^{2}} B\right) d t+\frac{\partial f}{\partial x} B d W$.

Note the unconventional minus sign. Similar change can be demonstrated for functions of many variables. Finally, the forward FP equation, which corresponds to our SDE (B.17) can be deduced from the forward FP equation corresponding to (B.18). In the case of many variables it reads

$\partial_{T} P=-\partial_{i^{\prime}}\left(A_{i^{\prime}} P\right)+\frac{1}{2} \partial_{i^{\prime}}\left(B_{i^{\prime} j^{\prime}} B_{j^{\prime} k^{\prime}} \partial_{k^{\prime}} P\right)$, 
where summation over repeating indices is understood. The structure of the diffusion term is different from the FP equation for the Ito SDE,

$\partial_{T} P=-\partial_{i^{\prime}}\left(A_{i^{\prime}} P\right)+\frac{1}{2} \partial_{i^{\prime}} \partial_{j^{\prime}}\left(B_{i^{\prime} k^{\prime}} B_{k^{\prime} j^{\prime}} P\right)$,

and the FP equation for the Stratonovich SDE,

$\partial_{T} P=-\partial_{i^{\prime}}\left(A_{i^{\prime}} P\right)+\frac{1}{2} \partial_{i^{\prime}}\left[B_{i^{\prime} k^{\prime}} \partial_{j^{\prime}}\left(B_{j^{\prime} k^{\prime}} P\right)\right]$

As usual, in addition to the forward FP equations (B.21) - (B.23) there exist the corresponding backward FP equations. If we begin with the backward equation for the Ito SDE,

$\partial_{t} P=-A_{i} \partial_{i} P-\frac{1}{2} B_{i k} B_{k j}\left(\partial_{i} \partial_{j} P\right)$,

and use the connection formulae (B.13)-(B.15) we find our backward FP equation

$\partial_{t} P=-\partial_{i}\left(A_{i} P\right)-\frac{1}{2} \partial_{i}\left(B_{i j} B_{j k} \partial_{k} P\right)$,

and the "Stratonovich form" of the backward FP equation

$\partial_{t} P=-\partial_{i}\left(A_{i} P\right)-\frac{1}{2} B_{i k}\left(\partial_{i} B_{k j} \partial_{j} P\right)$.

In total there are 3 versions of SDEs and 6 FP-equations. This ends the presentation of forward convention in stochastic calculus. For a detailed analysis of forward, backward and symmetric stochastic integrals and further references see ${ }^{[27]}$. Obviously, there exists a whole family of calculi for different weights placed on initial and final points in (B.9)(B.11).

We are now in a position to classify $\mathrm{Eq}(2.7)$. Consider a designated time axis, which begins at $t$, proceeds into future up to $T$, and then returns back to present. Fig. 2 shows the corresponding $S$-trajectory and $X$-trajectory. They belong to different sections of the "folded" time axis.

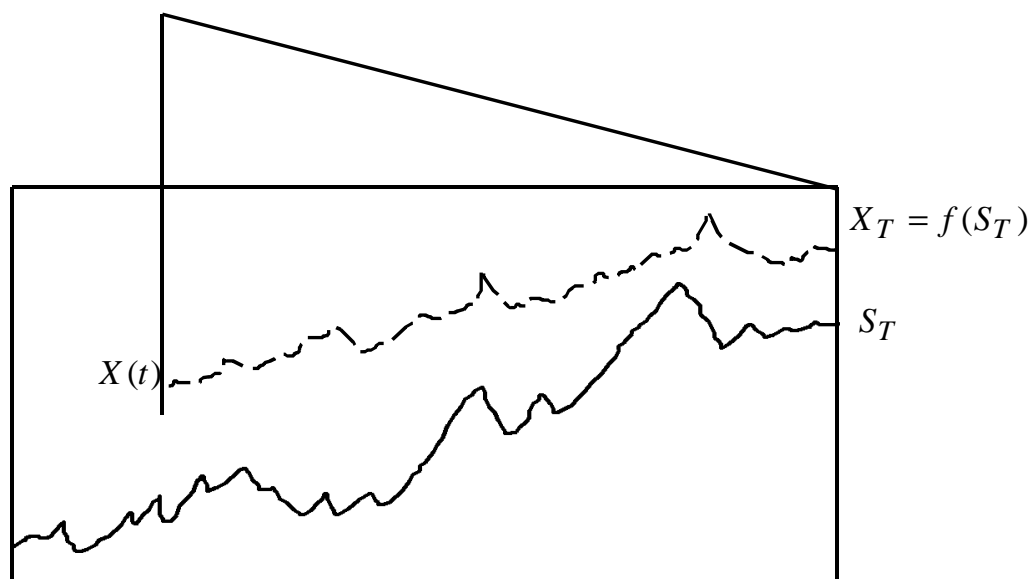




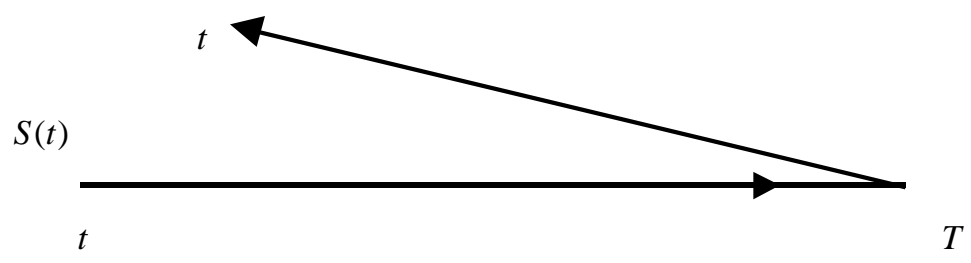

Fig.2 This "3D" illustration shows the "folded" time-axis (bold), corresponding to a contingent claim. Since the condition $X_{T}=f\left(S_{T}\right) \quad$ is imposed at the future moment $T$, the causality does not hold. Fluctuating lines show $S$-trajectory (solid) and $X$-trajectory (dashed).

Consider a "trajectory" which begins at $S(t)$ in the $S$-plane, proceeds into future to $S_{T}$, jumps to $X_{T}=f\left(S_{T}\right)$ at this moment, and then returns to $X(t)$ in the $X$-plane. Let us now define the probability of having $X(t)$ given that we begin with $S(t)$ along the "folded" time axis. When time $t$ decreases we simultaneously proceed "into the past" with our "initial" condition $S(t)$, and "into the future" with out target variable $X(t)$. That is why the resulting FP-equation looks like mixed forward \& backward. While the behavior of the "initial" condition is reflected by the corresponding terms of the backward I -equation, the behavior of the target variable is described by the terms of the forward E - equation. Using these guidelines and Eqs(B.23), (B.24), (B.1), (B.3) one can immediately compile $\mathrm{Eq}(2.7)$.

Eqs(2.7), (B.6), (B.7) are mathematically equivalent. Equivalence of (B.6) and (B.7) can be found in textbooks, e.g. ${ }^{[26]}$ page 56 . As for $\operatorname{Eq}(2.7)$ the probability $Q$ depends on $X^{\prime}$ and $X$ in the form $X^{\prime}-X e^{r(T-t)}$. Therefore, one can introduce a new variable $X^{\prime \prime}=X^{\prime} e^{-r(T-t)}-X$ in $\mathrm{Eq}(\mathrm{B} .7)$, which has the meaning of $\mathrm{P} \& \mathrm{~L}$ discounted to its present value, renormalize the probability density $Q$ to arrive to the probability density for $X^{\prime \prime}$ by using the multiplier $e^{r(T-t)}$, and integrate over $S^{\prime}$. For readers, who are familiar with $\mathrm{Eq}(\mathrm{B} .7)$, this gives the quickest derivation of $\mathrm{Eq}(2.7)$.

\section{Appendix C}

\section{Solutuions for Stop-Loss Hedging Strategies Based on Integral Transforms (by Alexander Lipton-Lifschitz,- Bankers Trust, NY)}

We consider the stop-loss strategy for the arithmetic Brownian motion. We introduce $\tau=\frac{1}{2} \sigma^{2}(T-t)$ and write $\operatorname{Eq}(4.1)$ as

$P_{\tau}=P_{S S}+\delta(S) P_{X}$, 
$P(X \mid S, 0)=\delta(X)$.

We combine the Fourier transform in $X$ and the Laplace transform in $\tau$ and rewrite the the problem (C.1), (C.2) in the form

$$
P_{S S}+[i k \delta(S)-\lambda] P=-1
$$

A simple algebra shows that

$$
P(k \mid S, \lambda)=\frac{1-\exp (-|S| \sqrt{\lambda})}{\lambda}-\frac{2 \exp (-|S| \sqrt{\lambda})}{\sqrt{\lambda}(i k-2 \sqrt{\lambda})} .
$$

The inverse Fourier transform yields

$$
P(X \mid S, \lambda)=\frac{1-\exp (-|S| \sqrt{\lambda})}{\lambda} \delta(X)+\frac{2 \exp [-\sqrt{\lambda}(|S|-2 X)]}{\sqrt{\lambda}} \theta(-X) .
$$

Finally, the inverse Laplace transform yields $\mathrm{Eq}(4.8)$ in the main text.

Now we show how to apply a similar technique in order to study the stop-loss strategy for the geometric Brownian motion. The problem corresponding to the strategy $\varphi=\theta(S-K)$ can be written as as

$$
P_{\tau}=P_{\xi \xi}-P_{\xi}+\delta(\xi) P_{x},
$$

$P(x \mid \xi, 0)=\delta(x)$,

where $\xi=\ln (S / K), x=\left[F+(S-K)^{+}\right] / K$. The Fourier-Laplace transform yields

$$
P_{\xi \xi}-P_{\xi}+[i k \delta(\xi)-\lambda] P=-1 \text {. }
$$

We write $P=Q e^{\xi / 2}+1 / \lambda$ where $Q$ is determined by the equation

$$
Q_{\xi \xi}+\left[i k \delta(\xi)-\lambda-\frac{1}{4}\right] Q=-\frac{i k \delta(\xi)}{\lambda},
$$

so that

$$
Q(k \mid \xi, \lambda)=-\frac{i k \exp (-|\xi| \sqrt{\lambda+1 / 4})}{\lambda(i k-2 \sqrt{\lambda+1 / 4})} .
$$

The inverse Fourier transform yields 


$$
Q(x \mid \xi, \lambda)=-\frac{\exp (-|\xi| \sqrt{\lambda+1 / 4})}{\lambda} \delta(x)+\frac{2 \sqrt{\lambda+1 / 4} \exp [-(|\xi|-2 x) \sqrt{\lambda+1 / 4}]}{\lambda} \theta(-x) .
$$

By virtue of the inverse Laplace transform after returning to the probability density we get

$$
\begin{aligned}
P(x \mid \xi, \tau)= & \left(1-\frac{|\xi| e^{\xi / 2}}{2} \int_{0}^{\tau} \frac{d \eta}{\sqrt{\pi \eta^{3}}} e^{-\xi^{2} / 4 \eta-\eta / 4}\right) \delta(x)+ \\
& +\frac{1}{2} \theta(-x) e^{\xi / 2} \int_{0}^{\tau} \frac{d \eta}{\sqrt{\pi \eta^{3}}} e^{-(|\xi|-2 x)^{2} / 4 \eta-\eta / 4} H_{2}\left(\frac{|\xi|-2 x}{2 \sqrt{\eta}}\right),
\end{aligned}
$$

where $H_{2}(z)=4 z^{2}-2$ is the Hermite polynomial. (C.12) is the resulting formula for the geometric Brownian motion. For the constant elasticity of variance problem we can obtain similar formulas but they are slightly more complicated and (naturally) involve the modified Bessel functions. 